\title{
Spin-correlated electronic state on the surface of a spin-orbit Mott system
}

\author{
Chang Liu, ${ }^{1}$ Su-Yang Xu, ${ }^{1}$ Nasser Alidoust, ${ }^{1}$ Tay-Rong Chang, ${ }^{2}$ Hsin Lin, ${ }^{3}$ Chetan Dhital, ${ }^{4}$ Sovit Khadka,${ }^{4}$ Madhab Neupane, ${ }^{1}$ \\ Ilya Belopolski, ${ }^{1}$ Gabriel Landolt, ${ }^{5}$ Horng-Tay Jeng, ${ }^{2}$ Robert S. Markiewicz, ${ }^{3}$ J. Hugo Dil,${ }^{5,6}$ Arun Bansil, ${ }^{3}$ \\ Stephen D. Wilson, ${ }^{4}$ and M. Zahid Hasan ${ }^{1}$ \\ ${ }^{1}$ Joseph Henry Laboratory and Department of Physics, Princeton University, Princeton, New Jersey 08544, USA \\ ${ }^{2}$ Department of Physics, National Tsing Hua University, Hsinchu 30013, Taiwan \\ ${ }^{3}$ Department of Physics, Northeastern University, Boston, Massachusetts 02115, USA \\ ${ }^{4}$ Department of Physics, Boston College, Chestnut Hill, Massachusetts 02467, USA \\ ${ }^{5}$ Swiss Light Source, Paul Scherrer Institut, CH-5232 Villigen, Switzerland \\ ${ }^{6}$ Physik-Institut, Universität Zürich-Irchel, 8057 Zürich, Switzerland
}

(Received 24 March 2014; revised manuscript received 25 June 2014; published 22 July 2014)

\begin{abstract}
We use angle-resolved photoemission spectroscopy to show that the near-surface electronic structure of a bulk insulating iridate $\mathrm{Sr}_{3} \mathrm{Ir}_{2} \mathrm{O}_{7}$ lying near a metal-Mott insulator transition exhibits weak metallicity signified by finite electronic spectral weight at the Fermi level. The surface electrons exhibit a spin structure resulting from an interplay of spin-orbit, Coulomb interaction and surface quantum magnetism. Our results shed light on understanding the exotic quantum entanglement and transport phenomena in iridate-based oxide devices.
\end{abstract}

DOI: 10.1103/PhysRevB.90.045127

PACS number(s): 71.27.+a, 71.30.+h, 74.25.Jb

\section{INTRODUCTION}

Strongly correlated electronic behavior can be modified near surfaces and interfaces of transition-metal oxides, leading to novel quantum phenomena [1-3]. Surfaces are known to be dramatically modified in topological band insulators and in spin-orbit coupled Rashba semiconductors [4-6]. These unusual surface effects not only reflect novel physics but also hold potential for future devices where such effects are amplified by nanostructuring, which leads to the enhancement of the surface-to-bulk ratio [7]. Recently, attention has focused on materials in which Mott physics and strong spin-orbit interaction may coexist in the bulk. Iridium oxides (iridates) have been identified as one of such promising classes of materials [8-17], in which the research has largely focused on the bulk properties. Theoretical models suggest the possibility of realizing exotic surface phenomena in the iridates, such as the arced semimetal [18], topological insulator [19], and high-temperature superconductivity [20], none of which has yet been found experimentally. These surface phenomena are often expected to be enhanced in correlated systems near the bulk metal-Mott insulator transition at which Coulomb interaction, spin-orbit coupling, and the often-frustrated magnetic moments compete in determining the ground state. The iridate we focus on here, $\mathrm{Sr}_{3} \mathrm{Ir}_{2} \mathrm{O}_{7}$, belongs to the Ruddlesden-Popper series whose bulk electronic structure lies in between a Mott insulator $(n=1)$ and a correlated metal $(n=\infty)$ [8,9], in the vicinity of a bulk criticality. Evidently, it is important to identify the surface ground state of such exotic iridates if they differ from the bulk.

In this paper, we report a systematic study of spin-integrated and spin-resolved angle-resolved photoemission spectroscopy (ARPES) to investigate the near-surface electronic structure of $\mathrm{Sr}_{3} \mathrm{Ir}_{2} \mathrm{O}_{7}$. While the bulk exhibits strongly insulating transport properties in this compound, our photoemission results on high-quality, single-phase $\mathrm{Sr}_{3} \mathrm{Ir}_{2} \mathrm{O}_{7}$ crystals reveal finite spectral weight at the Fermi level and a gaplike suppression for quasiparticles within $40 \mathrm{meV}$ of the Fermi level. In addition, the low-energy electrons exhibit strong left-right imbalanced modulation related to surface spin polarization, as well as a spin fine structure on one of the bands which indicates its in-plane spin polarization. Such a rich character of the surface ground state is not expected within the calculated and predicted bulk electronic structure. This material thus exhibits an interplay of spin-orbit coupling, antiferromagnetism, and surface termination, shedding light on understanding the exotic quantum entanglement and transport phenomena in the iridates.

\section{MATERIALS AND METHODS}

The high-quality single crystals of $\mathrm{Sr}_{3} \mathrm{Ir}_{2} \mathrm{O}_{7}$ used in this study were grown by a systematic self-flux method [8]. Ultraviolet spin-integrated ARPES measurements were performed at Beamline 4.0.3 (MERLIN) and 10.0.1 (HERS) of the Advanced Light Source (ALS), and Beamline 5-4 of the Stanford Synchrotron Radiation Lightsource (SSRL), using VG-Scienta R8000 and R4000 electron analyzers with incident photon energies ranging from 25 to $50 \mathrm{eV}$. Spin-resolved ARPES measurements were performed at the SIS-COPHEE Beamline at the Swiss Light Source (SLS), Paul Scherrer Institut. A setup with two mini-Mott spin detectors is utilized in the spin-resolved ARPES experiments, enabling simultaneous measurements of spin-polarization curves along all three $k$-space directions. The spin-resolved momentum distribution curve (MDC) mode at a binding energy of $\sim 50 \mathrm{meV}$ is used. Circular dichroic ARPES measurements were performed at the APPLE-PGM Beamline at the Synchrotron Radiation Center, Stoughton, Wisconsin, equipped with a Scienta $200 \mathrm{U}$ electron analyzer. Samples were cleaved in situ under a vacuum condition better than $4 \times 10^{-11}$ torr. Samples are found to be stable and without degradation for a typical measurement period of 24 hours. No charging effect due to insulating behavior was found for all $(\sim 15)$ samples measured.

Our first-principles band calculations are based on the generalized gradient approximation (GGA) [21] using the full-potential projected augmented wave method [22] as implemented in the VASP package [23]. In the calculation, spin-orbit coupling is included in the self-consistent cycles 
with 162 Monkhorst-Pack $k$ points sampling the Brillouin zone. The electron-electron interaction between the correlated $d$ electrons on Ir atoms is treated at the GGA $+U$ mean-field level. The value of $U$ is chosen to be $1.5 \mathrm{eV}$ and the strength of the spin-orbit coupling is artificially enhanced to be 1.7 times the original self-consistent value to match experimental observations. A simplified tetragonal structural model is utilized with in-plane lattice constant $a^{\prime}=a / \sqrt{2} \sim 3.90 \AA$. The long-range antiferromagnetic order is being considered by adding an antiferromagnetic zone boundary (AFZB) linking two $X$ points of the original Brillouin zone (i.e., a $\sqrt{2} \times \sqrt{2}$ magnetic structure) [24,25]. In order to reveal the spin texture of the surface bands, we used a slab model in which six layers of $\mathrm{IrO}_{6}$ planes (1.5 unit cells) are sandwiched by the top and bottom vacuum layers. An external electric field of $0.02 \mathrm{eV} / \AA \cdot e$ in strength pointing to the $+z$ direction is used for introduction of spin-degeneracy lifting.

\section{CRYSTALLOGRAPHIC PROPERTIES AND TRANSPORT DATA OF $\mathrm{Sr}_{3} \mathrm{Ir}_{2} \mathrm{O}_{7}$}

Figure 1 presents the crystallographic information of $\mathrm{Sr}_{3} \mathrm{Ir}_{2} \mathrm{O}_{7}$ and results from resistivity measurements. $\mathrm{Sr}_{3} \mathrm{Ir}_{2} \mathrm{O}_{7}$ crystallizes in a nearly tetragonal [26], layered perovskite structure with a $5 d^{5}$ low-spin configuration for the $\mathrm{Ir}^{4+}$ ions [8]. The corner-sharing $\mathrm{IrO}_{6}$ octahedra experiences a Jahn-Tellertype, alternating $12^{\circ}$ rotation about the $c$ axis [8]. Recent $\mathrm{x}$-ray resonance scattering studies [25] showed evidence that the magnetic moments of the $\mathrm{Ir}^{4+}$ ions in the ground state arrange antiferromagnetically, following the sign of the octahedra rotation [Figs. 1(a) and 1(b)]. The direction of these

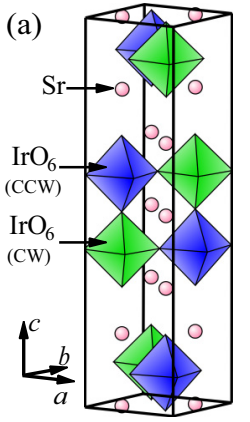

(b)

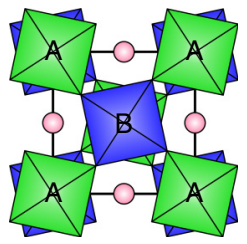

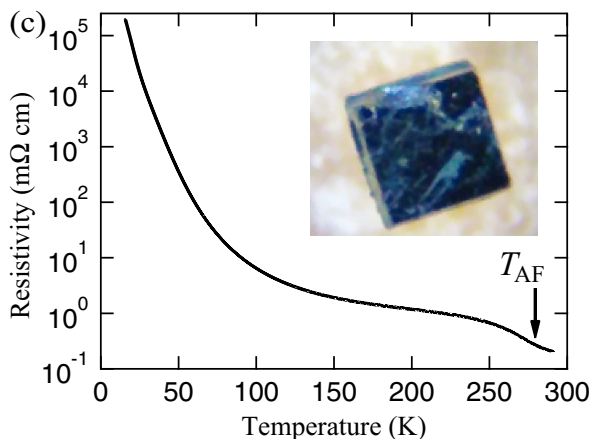

(d)

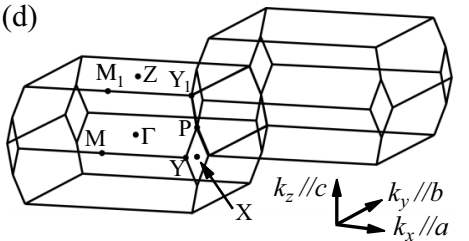

FIG. 1. (Color online) Crystallographic properties and resistivity data of $\mathrm{Sr}_{3} \mathrm{Ir}_{2} \mathrm{O}_{7}$. (a),(b) Crystal structure of $\mathrm{Sr}_{3} \mathrm{Ir}_{2} \mathrm{O}_{7}$. CCW (CW) represents the $12^{\circ}$ counterclockwise (clockwise) rotation of the $\mathrm{IrO}_{6}$ octahedra. A and B denote two different types of magnetic moment direction for the central Ir atom of each octahedra below $T_{\mathrm{AF}}$. (c) Resistivity curve of $\mathrm{Sr}_{3} \mathrm{Ir}_{2} \mathrm{O}_{7}$ up to $290 \mathrm{~K}$, showing clear insulating behavior. $T_{\mathrm{AF}} \sim 280 \mathrm{~K}$ marks the Néel temperature. Inset shows a picture of a typical $\mathrm{Sr}_{3} \mathrm{Ir}_{2} \mathrm{O}_{7}$ crystal. (d) Brillouin zone and notation of high-symmetry points of $\mathrm{Sr}_{3} \operatorname{Ir}_{2} \mathrm{O}_{7}$. magnetic moments is believed to be along the crystallographic $c$ axis, with an ordering wave vector $\mathbf{q}_{\text {Neel }}=(1 / 2,1 / 2,0)$ for temperatures below $T_{\mathrm{AF}} \sim 280 \mathrm{~K}$ [27]. If we consider the antiferromagnetic Brillouin zone (used throughout the paper) which is half the size of the paramagnetic zone, the Néel vector points to the $(1,0,0)$ direction. Figure 1 (c) shows the resistivity curve for a sample with high low-temperature resistivity. The differentiated in-plane resistivity curve as a function of temperature $\left[\frac{\partial \log \rho}{\partial(1 / T)}\right.$ vs $\left.T\right]$ is shown in Fig. 2(a). The antiferromagnetic transition temperature $T_{\mathrm{AF}}$ is clearly recognized by a kink in the $\rho-T$ curve. Quite surprisingly, results from surface-sensitive measurements such as scanning tunneling spectroscopy (STS) [28] and ARPES indicate a finite density of state at $E_{F}$ and thus a substantial electrical conductance down to the lowest measurable temperature. This controversy calls for our systematic ARPES study on the electronic structure of $\mathrm{Sr}_{3} \mathrm{Ir}_{2} \mathrm{O}_{7}$.

\section{RESULTS OF SPIN-INTEGRATED ARPES MEASUREMENTS}

Figure 2 shows the ARPES band dispersion data taken at $T=14 \mathrm{~K}$ within the $k_{x}-k_{y}$ plane [(001) plane]. At low temperatures, $\mathrm{Sr}_{3} \mathrm{Ir}_{2} \mathrm{O}_{7}$ changes from a paramagnetic phase to an antiferromagnetic (AF) phase, where the Ir moments point along the $c$ axis [29] with an in-plane commensurate Néel vector [25]. In Fig. 2(a), we plot the raw and differentiated in-plane resistivity as a function of temperature (adapted from Ref. [27]). The ordering temperature $T_{\mathrm{AF}} \sim 280 \mathrm{~K}$ is clearly shown as a sharp peak in the $\frac{\partial \log \rho}{\partial(1 / T)}$ vs $T$ curve [27]; a drastic upturn of the $\rho$ vs $T$ curve at low temperatures confirms its bulk insulating transport. Figures 2(b) and 2(c) summarize the in-plane electronic structure obtained from ARPES [Figs. 2(d) and 2(f)]. Two bands, $\alpha$ (blue) and $\beta$ (green), evolve to a close vicinity of the Fermi level. The shape and dispersive pattern of these low-lying bands are rather complicated. Figure 2(d) shows a typical ARPES Fermi mapping obtained with $35 \mathrm{eV}$ photons on the same batch of crystals we used for the resistivity measurements. Finite spectral weight is present at the Fermi level $\left(E_{F}\right)$, indicative of a nearly conductive ground state. The apparent bulk insulating behavior as well as the sharpness of the ARPES resolved bands show that the quality of our single crystals is as high as those used in other ARPES studies [11-13], ruling out the possibility that the weak metallicity we observed comes from impurity or other extrinsic effects. This finite intensity at $E_{F}$ can be due to a possible band bending effect close to the sample surface. Such a band bending effect-strong enough so that $E_{F}$ descends to near the bottom of the Mott gap at a depth greater than the electron escape depth-will give rise to an ARPES signal dominated by the more conductive surface layer. The fact that this conducting channel is not detected by conventional transport measurements may be due to a small surface/bulk volume ratio and difference in mobility between the bulkand surface-originated charge carriers. At $0.15 \mathrm{eV}$ binding energy [bottom panel of Fig. 2(e)], the $\alpha$ band decomposes into segments, and the $\beta$ band shrinks in size despite remaining intact. From this, we speculate that the $\beta$ band is electronlike, without ruling out other possibilities. In Fig. 2(f), we show 

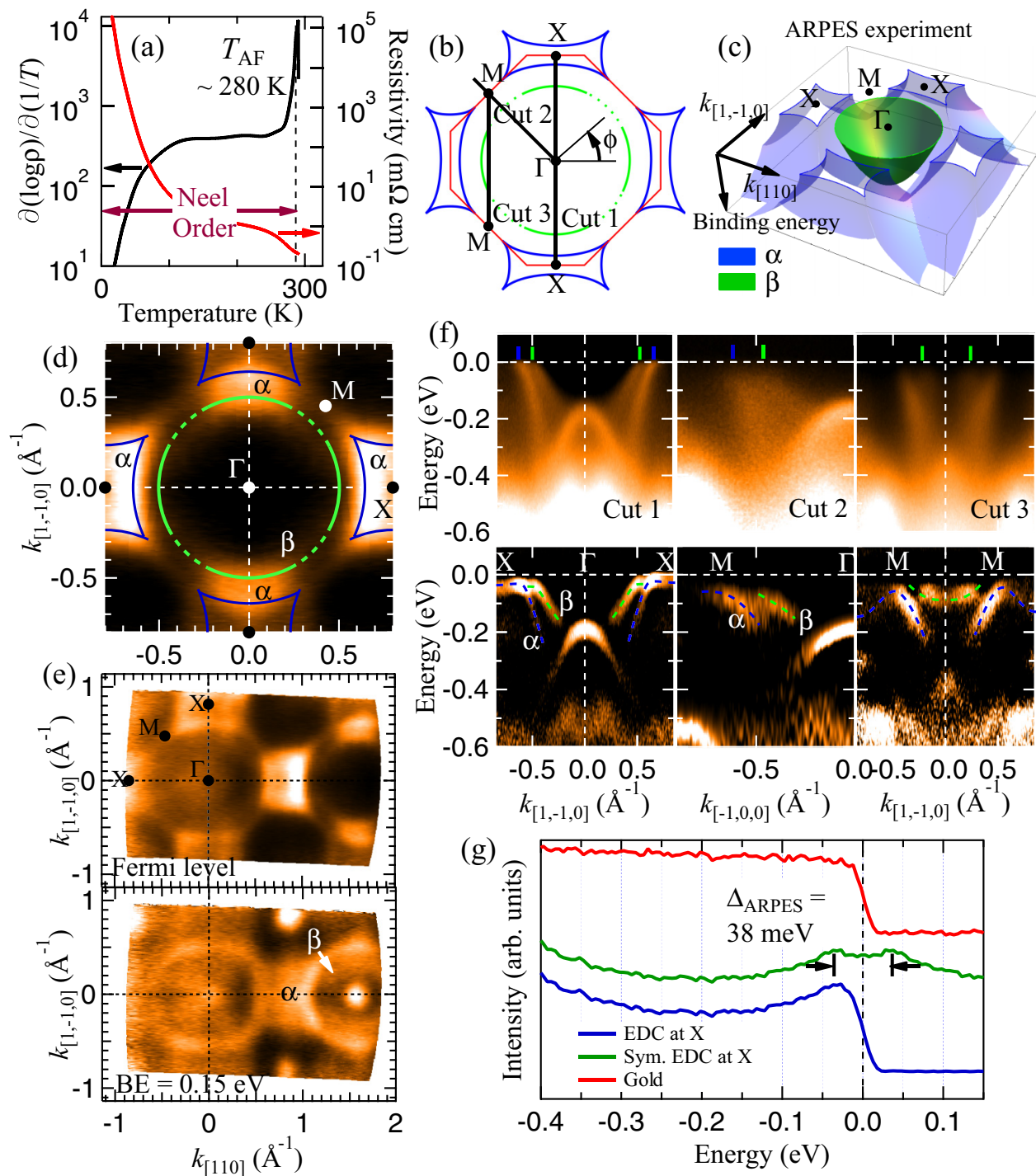

FIG. 2. (Color online) Surface electronic structure of $\mathrm{Sr}_{3} \mathrm{Ir}_{2} \mathrm{O}_{7}$. ARPES measurements are carried out at $14 \mathrm{~K}$ with 35 eV photons. (a) Temperature dependence of the raw $a-b$ plane resistivity $\rho$ (right axis) and differentiated $a-b$ plane resistivity $\frac{\partial \log \rho}{\partial(1 / T)}$ (left axis) for $\mathrm{Sr}_{3} \mathrm{Ir}_{2} \mathrm{O}_{7}$ [27]. (b) Sketch of ARPES constant energy map close to $E_{F}$. Red lines mark the in-plane bulk Brillouin zone at $k_{z}=0$. (c) Sketch of the experiment-derived in-plane band dispersion at $k_{z}=0$. (d) ARPES Fermi mapping [raw data in (e)]. Data is twofold symmetrized with respect to $k_{[110]}=0$. Blue and green colors represent the $\alpha$ and $\beta$ bands, respectively. (e) Raw ARPES mappings at 0 and 0.15 eV binding energies. (f) Band dispersion along three $k-E$ cuts shown in (b). Top: raw data; bottom: second derivative of raw data along the EDCs. (g) Analysis of the gap value at $X$. The two-peak structure of symmetrized EDC at $X$ near $E_{F}$ demonstrates the existence of a gap $\Delta_{\text {ARPES }}(X) \sim 38 \mathrm{meV}$.

three $k-E$ maps along directions shown in Fig. 2(b). The $\beta$ band is shown here to likely be an electronlike base due to the fact that this state shows an upward parabolic shape in all three $k-E$ cuts. Along the $\Gamma-X$ direction (cut 1 ), both the $\alpha$ state and the $\beta$ state are clearly resolved. Along the $\Gamma-M$ direction (cut 2 ), the $\beta$ band is shown to evolve to a lower binding energy near $M$, taking an upward parabolic shape. Along the $M-M$ direction (cut 3), the second derivative data also shows such upward parabolic behavior.

It should be noted from cuts 1 and 2 of Fig. 2(f) that despite the finite intensity at $E_{F}$, the low-energy quasiparticles experience a gaplike spectral weight suppression (SWS), signified by a gradual decrease of ARPES intensity as the bands approach the Fermi level [Fig. 2(g)], similar to other correlated oxides [30]. The second derivative analysis of cut 1 [31] (bottom panels) reveals that along the $\Gamma-X$ direction, the $\alpha$ band bends horizontally to form a van Hove-like flat portion (detailed in Fig. 5), while the $\beta$ band loses its intensity. Figure 2(g) shows the existence of the SWS at the $X$ point where the $\alpha$ band evolves closest to $E_{F}$ (i.e., top of the flat portion). The exact location of the Fermi level is obtained by fitting the polycrystalline gold data with the Fermi distribution function; the energy distribution curve (EDC) at the $X$ point is then symmetrized with respect to $E_{F}$. The two peak and valley line shape of the symmetrized EDC proves the presence of the SWS, which is about $38 \mathrm{meV}$ in size. This value is smaller than the full insulating gap value obtained from optical measurements ( $\sim 250 \mathrm{meV}$ [9]), indicative of a different $E_{F}$ 


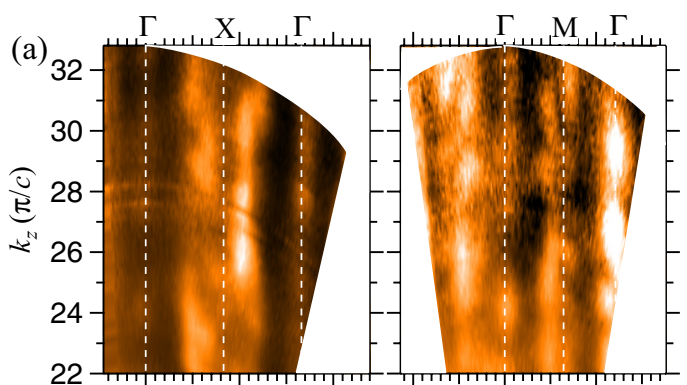

(b)
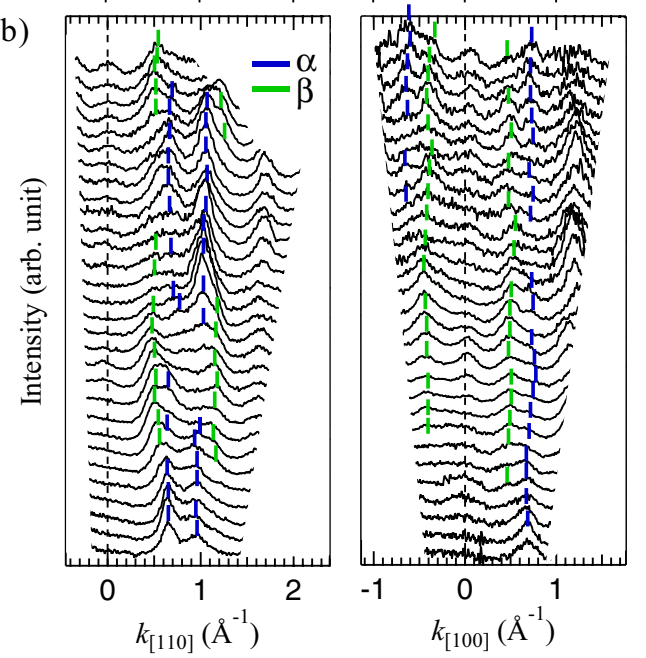

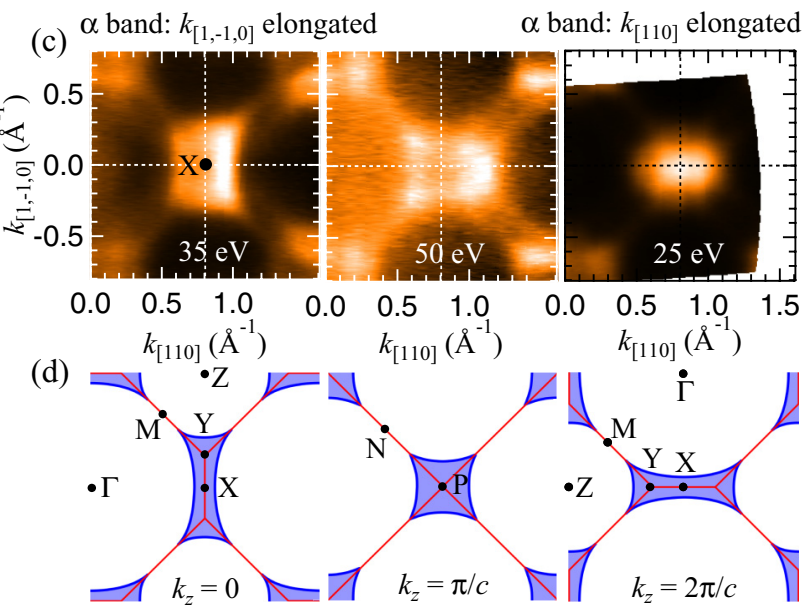

ARPES experiment

(f)

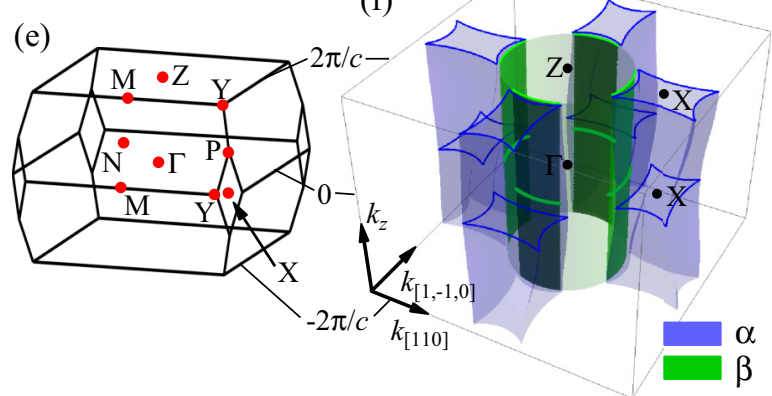

FIG. 3. (Color online) Quasi two dimensionality of the electronic states. (a) $k_{z}$ dispersion data $(40<h v<80 \mathrm{eV})$ along two high-symmetry directions. $k_{z}$ values are calculated using an inner potential $V_{0}=10 \mathrm{eV}$. (b) MDC stacks of the corresponding panels in (a). Blue and green bars mark the $\alpha$ and $\beta$ bands, respectively. (c) ARPES Fermi mappings with 35, 50, and $25 \mathrm{eV}$ photons, corresponding to $k_{z}$ values of $\sim 0, \sim \pi / c$, and $\sim 2 \pi / c$, respectively. (d) Sketches of experimental constant energy maps (CEMs) near $E_{F}$ for the corresponding photon energies in (c). (e) Brillouin zone and notation of high-symmetry points. (f) Schematics of the experiment-derived three-dimensional CEM.

at the crystal surface resulting from effective band bending. Importantly, this SWS is reproduced in our high-temperature data [Figs. 4(c) and 4(d)], with an energy scale of $\sim 35 \mathrm{meV}$, indicating again that such SWS is not due to impurity or other extrinsic effects (detailed below). Note that concurrent STS results [28] on the same samples used in our studies consistently support our observation that the surface Fermi level of $\mathrm{Sr}_{3} \mathrm{Ir}_{2} \mathrm{O}_{7}$ always lies close to the top of the valence bands. To sum up our results in Fig. 2, we observe a gaplike suppression of spectral weight for the spin-orbit correlated electrons at the surface of $\operatorname{Sr}_{3} \operatorname{Ir}_{2} \mathrm{O}_{7}$ within a narrow energy window of $E_{F}$, as well as a Fermi contour (the $\beta$ band) that likely centers at $\Gamma$.

In Fig. 3, we show $k_{z}$ dispersion analysis for $\mathrm{Sr}_{3} \mathrm{Ir}_{2} \mathrm{O}_{7}$ with photon energies ranging from 25 to $80 \mathrm{eV}$. From the raw dispersive pattern and the associated momentum distribution curves (MDCs) [Figs. 3(a) and 3(b)], we find that the resolved bands show little $k_{z}$ dispersion, as both the $\alpha$ and $\beta$ bands form nearly vertical lines in the $k_{\|}-k_{z}$ plane along both the $\Gamma-X$ and $\Gamma-M$ directions, which is evident for a quasi-two-dimensional electronic structure. On the other hand, our detailed analysis shown in Figs. 3(c) and 3(d) reveals that the $\alpha$ band does show some extent of a periodic $k_{z}$ dispersive pattern, which is required by the symmetry of the AF Brillouin zone. In Fig. 3(d), we show that the $Y-X-Y$ segment of the AF zone edge changes from a vertical line at $k_{z}=0$ to a single $\operatorname{dot} P$ at $k_{z}=\pi / c$, and, finally, to a horizontal line at $k_{z}=2 \pi / c$. As a result, any band close to this segment will have to change from a $k_{[1,-1,0]}$ elongated shape at $k_{z}=0$ to a $k_{[110]}$ elongated shape at $k_{z}=2 \pi / c$. This is consistent with our ARPES results shown in Fig. 3(c), where the Fermi mappings done with $h v=35$, 50 , and $25 \mathrm{eV}$ roughly correspond to the situation at $k_{z}=0$, $\pi / c$, and $2 \pi / c$, respectively. To clarify our observations from Figs. 2 and 3, a schematic constant energy map close to the top of the $\alpha$ band (not at $E_{F}$ ) is presented in Fig. 3(f) associated with the AF Brillouin zone [Fig. 3(e)]. From this figure, one can see that although some weak $k_{z}$ dispersion is discernible, the electronic structure of $\mathrm{Sr}_{3} \mathrm{Ir}_{2} \mathrm{O}_{7}$ is mostly two dimensional. It is very important to note here that such finite $k_{z}$ dispersion is not excluded for surface-related bands, especially for systems with small insulating gaps, since the surface bands can, in principle, penetrate deeper into the bulk and thus respect the symmetry of bulk electrons. The spin textured behavior (Figs. 6 and 7) together with the observed weak $k_{z}$ dispersion in these states points away from the possibility that the $\alpha$ and $\beta$ bands are purely bulk bands. In our understanding, these bands may originate from the crystal bulk, but undergo important surface modifications.

To demonstrate the robustness of the surface-modified electronic structure in $\mathrm{Sr}_{3} \mathrm{Ir}_{2} \mathrm{O}_{7}$, ARPES measurements are 


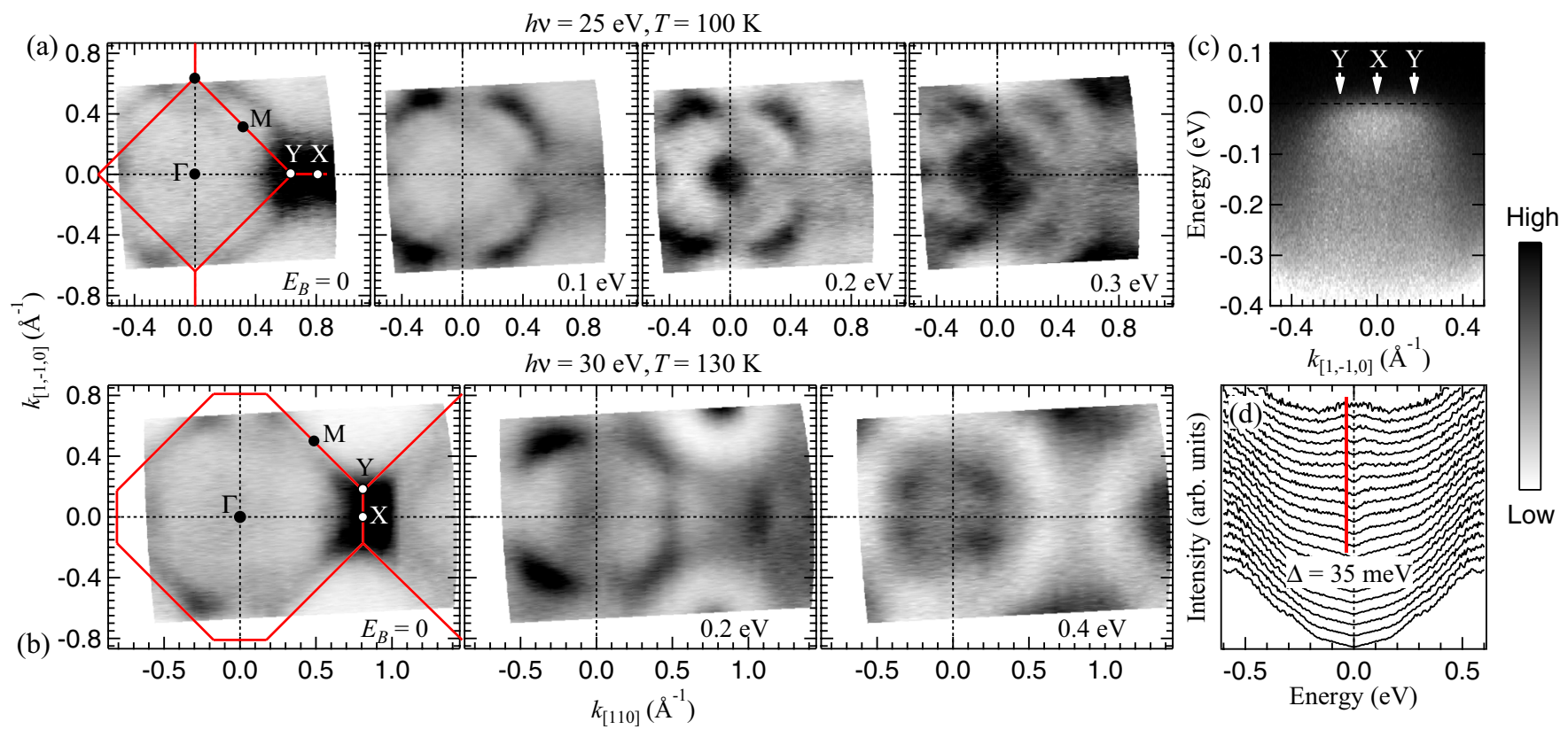

FIG. 4. (Color online) ARPES constant energy maps of $\mathrm{Sr}_{3} \mathrm{Ir}_{2} \mathrm{O}_{7}$ at elevated temperatures. Dark area represents higher intensity (see color scale). (a) ARPES constant energy maps at $T=100 \mathrm{~K}$, measured with $25 \mathrm{eV}$ photons. Binding energy $\left(E_{B}\right)$ for each map is marked at the lower right corner of each panel. (b) Same as (a), but at $T=130 \mathrm{~K}$, measured with $30 \mathrm{eV}$ photons. Red solid lines in (a) and (b) mark the Brillouin-zone boundaries. Note that the zone boundaries are different for 25 and $30 \mathrm{eV}$ maps (same as in Fig. 3). (c) $k-E$ map along the $Y$ - $X-Y$ direction for data in (b). (d) Symmetrized EDC stacks corresponding to data in (c). Red line indicates the top of the van Hove-like flat portion of the $\alpha$ band, showing a $\sim 35 \mathrm{meV}$ spectral weight suppression similar to Fig. 2(g).

performed at different temperatures for crystals from numerous batches. In Fig. 4, we present ARPES constant energy maps obtained from a different sample than that shown in Fig. 2. Measurement temperatures here are 100 and $130 \mathrm{~K}$, compared to $T=14 \mathrm{~K}$ for Fig. 2. It is clear from Fig. 4 that the bands approaching the Fermi level $\left(E_{F}\right)$ as well as weak metallicity (absence of electrical charging effect) persist at these elevated temperatures. As in Fig. 2, there are two visible bands at the vicinity of $E_{F}$ for both photon energies presented. The highest photoelectron intensity comes from the $\alpha$ band around the zone corner $X$ where it evolves to very low binding energies. The holelike nature of the $\alpha$ band and the likely electronlike nature of the $\beta$ band are indicated from their expansion and shrinkage at higher binding energies, respectively. The $k_{z}$ dependence of the $\alpha$ band (Fig. 3) is also apparent here, as the long axis of the rectangular $X$ contour changes from the $k_{[110]}$ direction at $25 \mathrm{eV}$ photons to the $k_{[1,-1,0]}$ direction at $30 \mathrm{eV}$ photons. Importantly, the $\sim 40 \mathrm{meV}$ SWS seen at low temperature [Fig. 2(g)] can also be resolved at high temperatures. In Figs. 4(c) and 4(d), we present a $k-E$ map along the $Y-X-Y$ direction where the $\alpha$ band evolves closest to $E_{F}$, together with the corresponding symmetrized EDC stacks. Similar to the case in Fig. 2, we see that all symmetric EDCs exhibit a peak-dip-peak line shape across $E_{F}$, signifying a nonzero gaplike SWS of $\sim 35 \mathrm{meV}$, a value very close to the one at low temperatures [Fig. 2(g)]. Such reproducibility proves that the surface electronic structure, especially the gaplike SWS of $\sim 40 \mathrm{meV}$ at the Fermi level, remains unchanged at elevated temperatures if we choose to study only the low-resistivity samples. $\mathrm{Sr}_{3} \mathrm{Ir}_{2} \mathrm{O}_{7}$ is thus a well-established insulator having a marginally conducting surface layer up to intermediate temperatures.

In Fig. 2(f), we plotted an ARPES $k-E$ map along the $X-\Gamma-X$ direction (cut 1 ), showing a plateaulike flat portion of bands around the zone corner $X$. Here we present a detailed analysis of this flat portion. Figure 5 shows consecutive $k-E$ cuts across the $X$ region [Fig. 5(a)] along with corresponding energy distribution curves (EDCs) [Fig. 5(b)]. The most important observation from these EDCs is an intensity peak located at $\lesssim 40 \mathrm{meV}$ below the Fermi level. This feature shows little visible dispersion at the circumference of a rectangular-shaped $k$-space region covering an area of about $0.8 \times 0.6 \pi^{2} / a^{2}$, while it experiences a reduction in intensity inside this region (i.e., close to the $X$ point; see data for cuts 3-5). Therefore, such intensity peaks form a plateaulike flat portion, the top of which locates very close to $E_{F}$ but does not cross $E_{F}$. In our first-principles band calculations (Fig. 8), this flat portion is nicely reproduced when assuming the existence of an antiferromagnetic zone boundary (AFZB) linking two $X$ points of the original Brillouin zone, as in the case of the iron arsenic superconductors [32], although detail differences in binding energies between calculation and experiment point to a surface band bending effect. It should be noted here that the presence of an AFZB serves as evidence for an antiferromagnetic ground state with a Néel vector $\mathbf{q}_{\text {Neel }}=(1 / 2,1 / 2,0)$ [equivalent to $(1,0,0)$ in the AF Brillouin zone with two iridium atoms per unit cell] at low temperatures. Moreover, the resulting enhanced density of states in close vicinity to $E_{F}$ points to a governing role that the $\alpha$ band plays in explaining data from surface thermal transport measurements. In Fig. 5(d), we study the spectral weight caused by this feature 

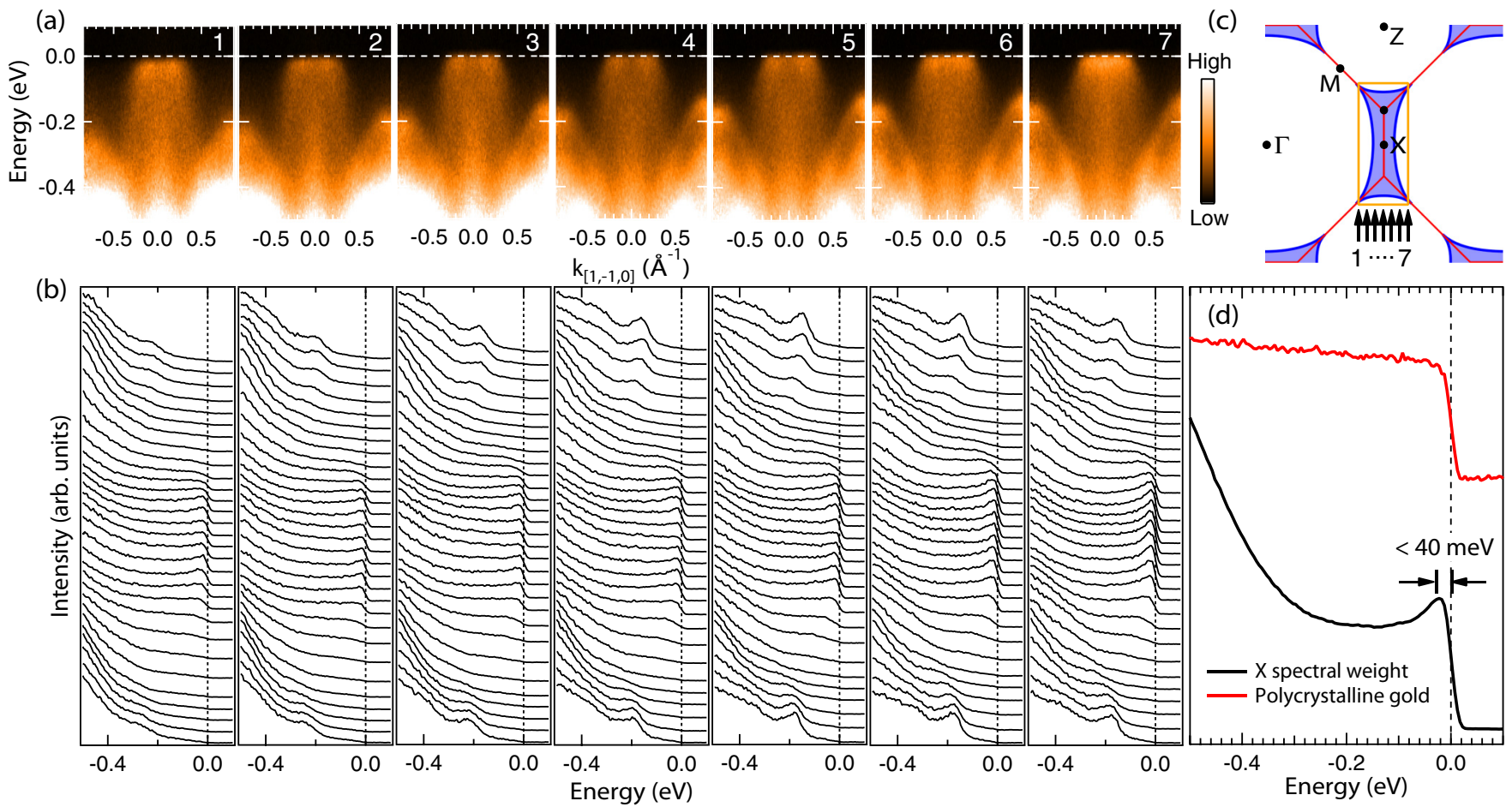

FIG. 5. (Color online) Detailed analysis for the plateaulike flat portion for bands around the zone corner $X$. Data is taken with 35 eV photons at $T=14 \mathrm{~K}$. (a) A series of ARPES $k-E$ maps near $X$. $k$-space locations of these maps (cuts 1-7) are marked by black arrows in (c). (b) Energy distribution curves (EDCs) for the corresponding map in (a). Note that the $X$ bands evolve to binding energies as low as $\sim 40$ meV. (c) Sketch of the observed Fermi contours around $X$. (d) Black curve: Spectral weight at the zone corner-integration of EDCs within the $k$-space range given by an orange box in (c). Red curve: data of a polycrystalline gold sample in electrical contact with the $\mathrm{Sr}_{3} \mathrm{Ir}_{2} \mathrm{O}_{7}$ crystal, showing the exact location of $E_{F}$. Note that the energy scale for the spectral weight peak just below $E_{F}$ comes from the flat portion of the $X$ bands.

via an integration of the EDCs within the rectangular region around $X$ [orange box in Fig. 5(c)]. The enhanced spectral weight signified by an intensity peak is clearly visible at binding energy $\sim 40 \mathrm{meV}$. Figure $2(\mathrm{~g})$ duplicates the curve in Fig. 5(d). A future systematic research on the temperature evolution of this spectral weight would serve as a helpful guide for the recognition of the surface conducting signal from thermoelectric measurements.

We remark here that some degree of cleavage (growth batch) dependence is seen on the electronic structure of $\mathrm{Sr}_{3} \mathrm{Ir}_{2} \mathrm{O}_{7}$. In particular, the energy scale of the gaplike SWS is found to have minor sample-to-sample modulations, and the crystallinity of each sample is found to be different, signified by different sharpness of the resolved bands. Despite the different SWS energy scale, no sample is found to be metallic (bands crossing $E_{F}$ ) or " $n$ type." Also, the ARPES data on the samples that have slightly larger SWS sizes are much broader (larger peak width for bands) than those shown in Figs. 2-5, indicative of poorer crystallinity. Based on this fact and the criteria mentioned above for choice of samples, data from other sets of samples are not included in the paper.

\section{RESULTS OF SPIN-RESOLVED ARPES MEASUREMENTS}

In Fig. 6, we present the spin-resolved (SR) ARPES measurements of $\mathrm{Sr}_{3} \mathrm{Ir}_{2} \mathrm{O}_{7}$. A spin-integrated ARPES $k$ - $E$ map along the $X-\Gamma-X$ direction [see also Fig. 2(f)] is shown in Fig. 6(a) for clarification of momentum-space positions and band notations. The important observations of this data set are (1) the strong left-right imbalance of the SR-ARPES signal and (2) the spin fine structure of the $\beta$ band. We summarize our results in Fig. 6(b), where the in-plane spin-polarization angles at different $k$ points are presented [33] [numbers in Fig. 6(b)]. It is seen from Fig. 6(b) that both the $\alpha$ and the $\beta$ band are spin polarized. The integrated spin for the $\alpha$ band near the left and the right $X$ momenta point to opposite directions. The $\beta$ band likely consists of two close-by rings with in-plane spin helicity. In Figs. 6(c)-6(e), we show the spin-polarization analysis for a MDC cut along the $X-\Gamma-X$ direction at $\sim 50 \mathrm{meV}$ binding energy, within the same energy window as the SWS observed in Fig. 2. The spin direction under study is along $[1,-1,0]$, which is tangential to the $\beta$ contour. First, multiple peaks are present in the total intensity curve (blue circles). The spin-up and spin-down components show strong antisymmetry with respect to the zone center $\Gamma$. Since this behavior contradicts the Kramers' theorem, one possible reason for its occurrence is the presence of surface antiferromagnetism (AFM) which explicitly breaks time-reversal symmetry, although we cannot rule out other many-body effects which give rise to a collective net spin polarization at the surface. Moreover, the matrix element effect can also result in the observed signal asymmetry. These effects introduce a spin-polarized background; spin-polarized 

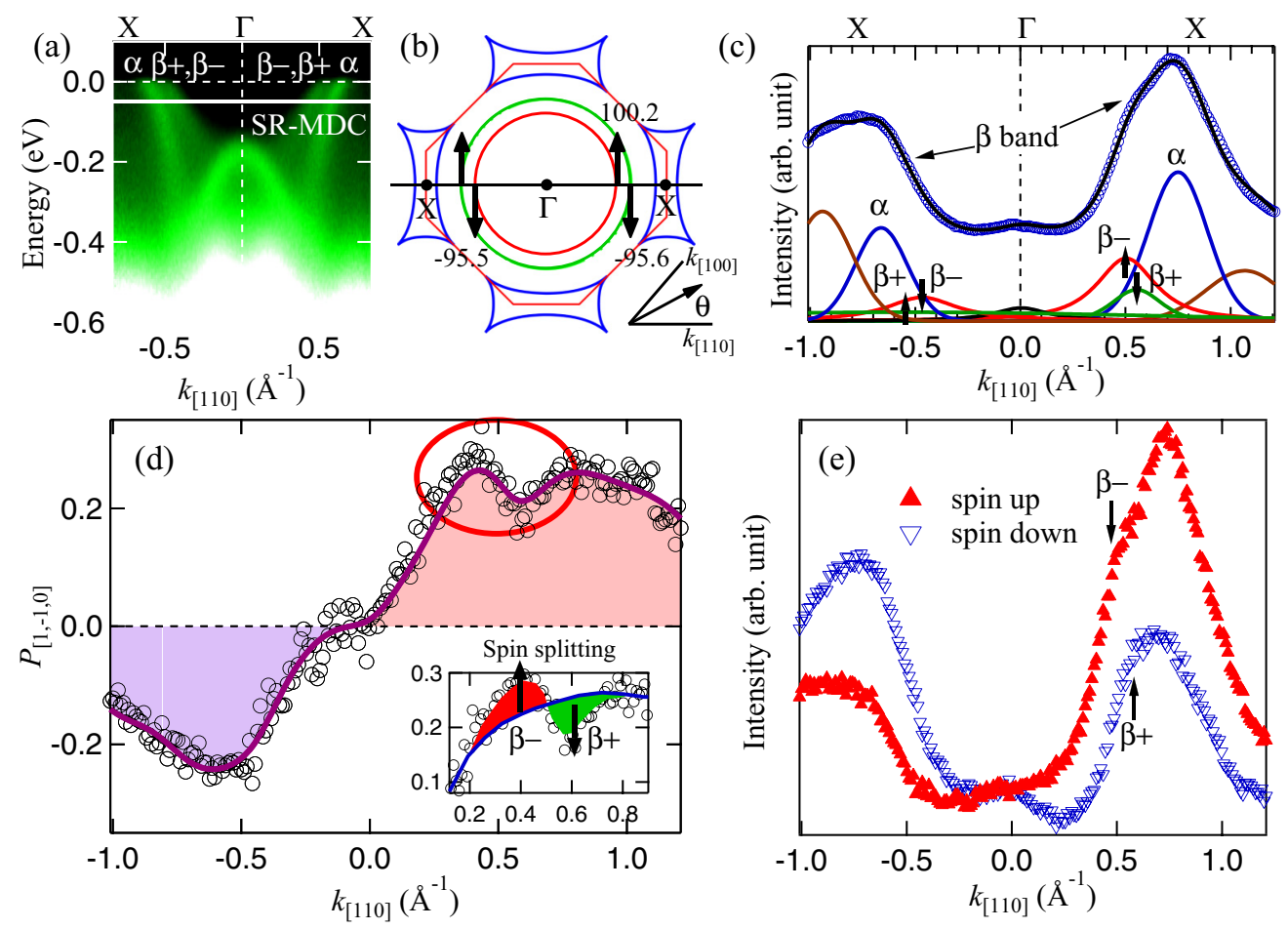

FIG. 6. (Color online) Spin polarization of electrons on the surface. (a) ARPES $k$ - $E$ map along the $\Gamma-X$ direction. White solid line denotes the spin-resolved MDC studied in (c)-(e). (b) Summary of results from spin-resolved ARPES measurements, together with notations of the AF Brillouin zone and cut direction in (b)-(d). Blue contours represent the projected $\alpha$ band. Black arrows represent the Rashba-like in-plane spin-polarization direction; numbers represent the experimental in-plane spin-polarization angles $(\theta)$. (c) Spin-integrated ARPES intensity along $\Gamma-X$ at a binding energy of $\sim 50 \mathrm{meV}$. Data is taken at $T=20 \mathrm{~K}$ with $35 \mathrm{eV}$ photons. Lower curves represent the multiple Lorentzian fit of the spin-integrated curve. Black curve is the sum of all Lorentzians. (d) Polarization curve for spin component $S_{[1,-1,0]}$ along $\Gamma-X$. Insets show the Rashba-like in-plane spin fine structure of the $\beta$ band. (e) Spin-resolved ARPES intensity along the same direction as in (d). Arrows and symbols mark the apparent "shoulders" in the spin-polarized intensity that corresponds to the $\beta-$ and $\beta+$ bands in (d).

signals from individual bands sit on top of this background. Determination of the background curve [blue curve in the inset of Fig. 6(d)] is based on the assumption that the background should be smooth across the corresponding $k$ range, while rapid changes of spin polarization are the results of spin polarization of individual bands. Second, the spin fine structure of the $\beta$ band is observed in the raw polarization curve [Fig. 6(d)]. At around $k=0.5 \AA^{-1}$, the $P_{[1,-1,0]}$ polarization curve first increases and then decreases within a narrow $k$ range (red ellipse). The inner upturn $\left(k \sim 0.48 \AA^{-1}\right)$ originates from a higher spin-up intensity, while the outer downturn $(k \sim$ $0.55 \AA^{-1}$ ) originates from a higher spin-down intensity [inset of Fig. 6(d)]. The spin splitting of the $\beta$ band is better resolved in Fig. 6(e), where arrows and symbols mark the apparent "shoulders" in the spin-polarized intensity that corresponds to the $\beta-$ and $\beta+$ bands in Fig. 6(d). It is important to point out here that no matter what fitting procedure we use, these signatures for spin splitting of the $\beta$ band always appear, which supports our statement of a fine spin split for the $\beta$ band. The direction of spin polarization for the two rings is obtained from simultaneous data fitting for all observable peaks of the spin-resolved MDC as well as the spin-integrated MDC, a procedure which is independent from the choice of the background curve. Momentum splitting for the two spin components is determined to be $\Delta k_{[110]} \sim 0.063 \AA^{-1}$. Note that the $\beta$ band spin splitting to the left of $\Gamma$ is not apparent in the raw polarization curve. Although the simultaneous peak fitting analysis succeeded in revealing the in-plane spin direction for the inner contour to the left of $\Gamma$ [number in Fig. 6(b)], this does set some limitation to our discussion on the "Rashba-like" spin configuration. The fine spin texture of the $\beta$ band should be resolved by future experiments.

In addition to the spin polarization along $P_{[1,-1,0]}$ as shown in Fig. 6(e), in Fig. 7 we present the complete spin-resolved ARPES data set along the $X-\Gamma-X$ momentum cut. Data is taken at $20 \mathrm{~K}$ with $35 \mathrm{eV}$ photons, polarized parallel to the scattering plane ( $\pi$ configuration). The angle between the incident light and the sample surface normal is set at $45^{\circ}$. It is seen from Fig. 7 that significant spin polarization occurs not only in the $[1,-1,0]$ directions, but also in the [110] and [001] directions. Spin polarization in the [110] direction is roughly symmetric with respect to $\Gamma$. This indicates a tilting of the $\alpha$ (and/or $\beta$ ) band spin direction towards $\Gamma-X$, which does not affect our discussion for the $\beta$ band spin direction. The $P_{z}$ curve also experiences a rapid upturn at around $k=0.5 \AA^{-1}$. Similar to the $P_{[1,-1,0]}$ analysis of Fig. 6 , we attribute this upturn to a spin component pointing outside the sample surface for the outer $\beta$ contour. Note that a small $P_{z}$ upturn is also visible at $k=-0.5 \AA^{-1}$, indicative of upward spin direction for the entire outer $\beta$ contour. Such out-of-plane spin fine modulation is consistent with a theoretical model where finite Coulomb 

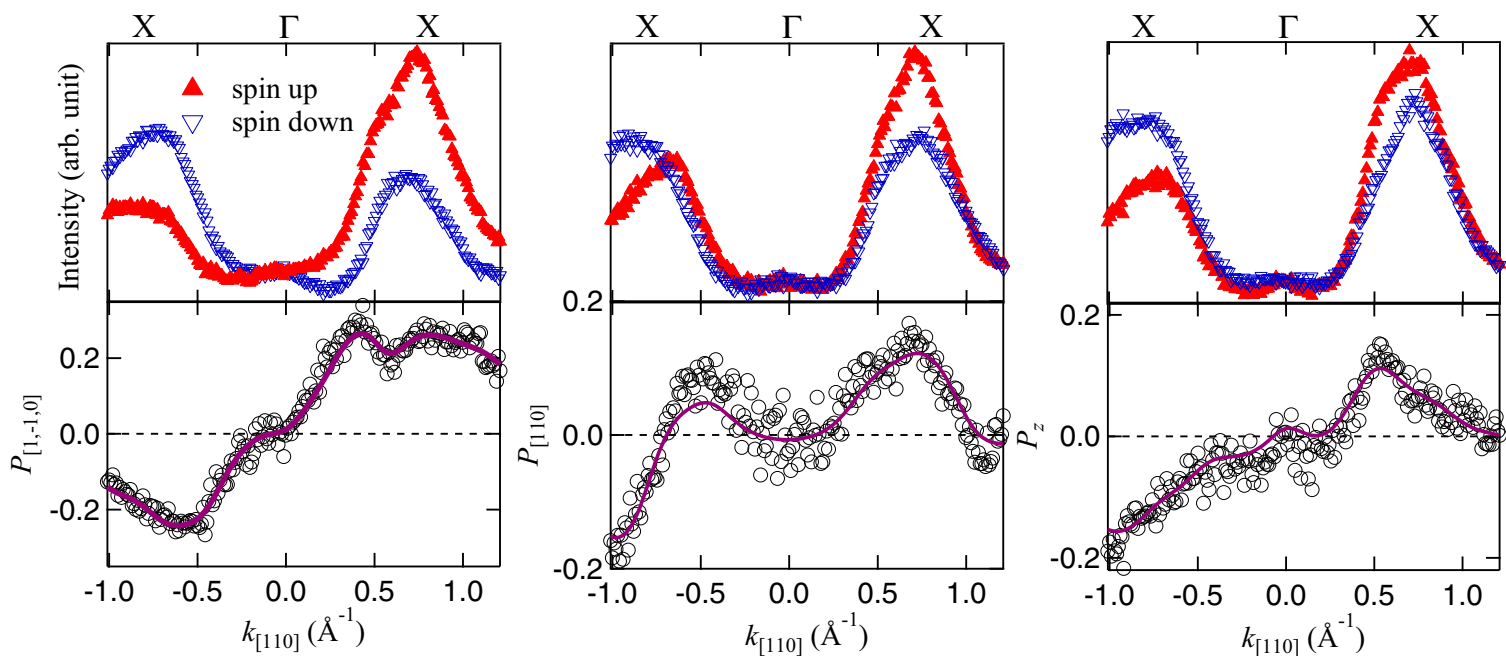

FIG. 7. (Color online) Complete spin-resolved ARPES data set along $X-\Gamma-X$. Top: Spin-resolved ARPES intensity for three spin components, $S_{[1,-1,0]}, S_{[110]}$, and $S_{z}$, along $X-\Gamma-X$. Bottom: Corresponding spin-polarization curves.

$U$ and surface antiferromagnetism give rise to Rashba-like in-plane spin texture, together with Zeeman-like out-of-plane spin split, although we cannot rule out other many-body effects which give rise to a collective out-of-plane spin polarization at the surface.

Such surface spin modulation is previously unobserved for a system with both strong spin-orbit coupling and onsite
Coulomb interaction. These spin-correlated surface electronic states are not observed in the high- $T_{c}$ superconducting cuprates, which indicates strong dissimilarity of the surfacemodified electronic states between the high- $T_{c}$ superconductors and the layered iridates, unlike what is predicted from theory [20]. Our finding adds an additional variable (or tunability) to the electronic phase diagram of the iridates.

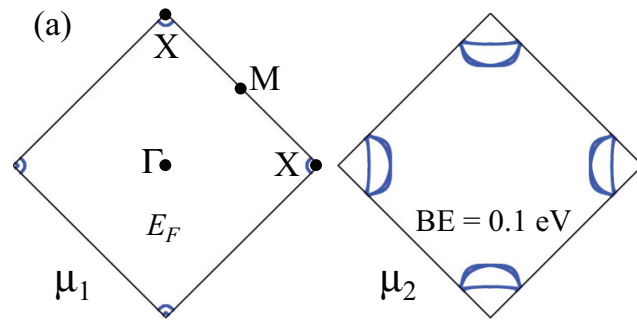

Bulk band structure

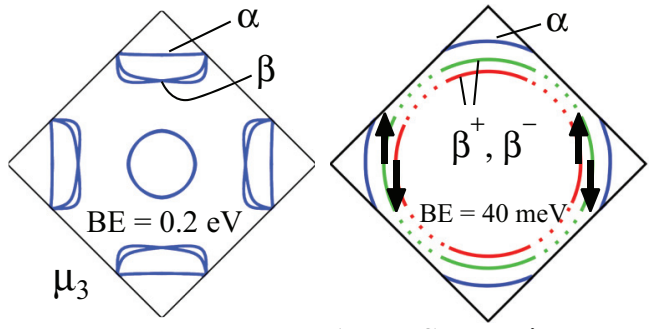

ARPES experiment (b)

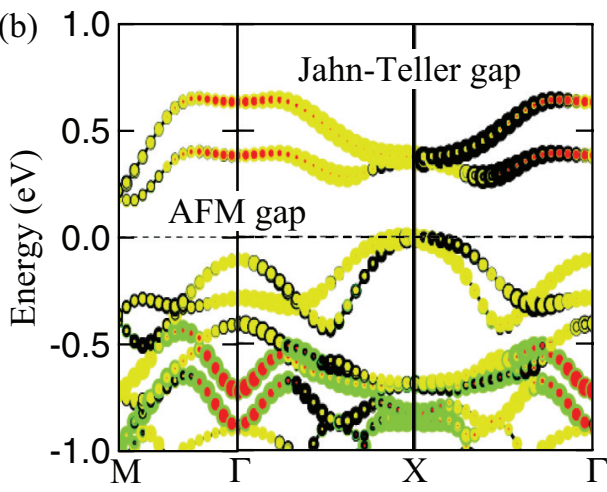

Bulk state: (c)

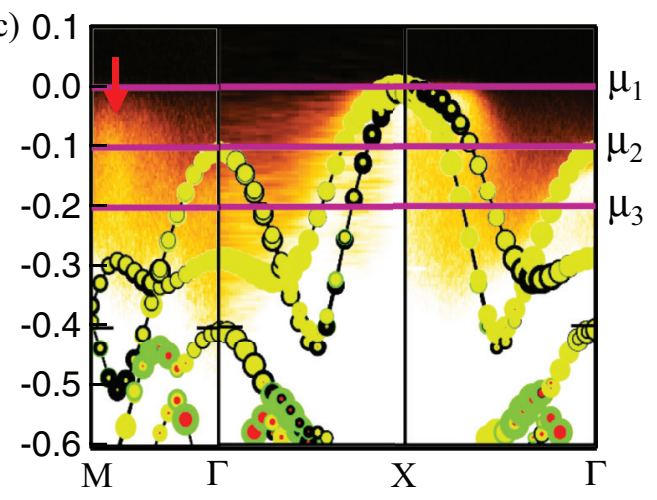

$5 d_{3 z^{2}-r^{2}} \bigcirc 5 d_{x^{2}-y^{2}} \equiv$ Fermi surfaces (expt.)

FIG. 8. (Color online) Layered iridate GGA $+U$ theory vs ARPES experiment. (a) Calculated constant energy maps (CEMs) at different binding energies, indicated by purple lines in (c), vs the experimentally derived CEM at $E_{B} \sim 40 \mathrm{meV}$ (lower right panel). Black arrows show the Rashba-like fine texture of spin detected for the $\beta$ band. (b) Results of band calculation (detailed in text). Solid circles with different colors and sizes represent the contribution of different Ir $5 d$ orbitals. (c) Band calculation on top of ARPES band structure close to $E_{F}$ (same data set as Figs. 2 and 3). Red arrow points to the $\beta$ band approaching $E_{F}$ near $M$ that is not present in the calculation. 
Since such surface-related behavior is not observed in $\mathrm{Sr}_{2} \mathrm{IrO}_{4}$ or $\mathrm{SrIrO}_{3}$, it is likely that the proximity to the bulk criticality contributes to the surface modification.

\section{RESULTS OF GGA BAND CALCULATIONS AND COMPARISON WITH ARPES DATA}

In Fig. 8, we present first-principles $\mathrm{GGA}+U$ band calculations that reveal a bulk electronic structure agreeing reasonably well with our measurements for high binding energies. In order to achieve such agreement, we set the Hubbard $U=1.5 \mathrm{eV}$ and $\lambda_{\mathrm{SO}}=1.7$ times the self-consistent value in the calculation. Therefore, our data places constraints on the magnitude of Coulomb $U$ and spin-orbit coupling experimentally realized in the material under study. The $12^{\circ}$ rotation of the $\mathrm{IrO}_{6}$ octahedra (Fig. 1) gives rise to a Jahn-Teller-type gap locating at $0.7-1.3 \mathrm{eV}$ above $E_{F}$. The combined effect of $U$ and $\lambda_{\text {SO }}$ causes the opening of a partial gap at $E_{F}$ close to $\Gamma$; this gap enlarges and becomes a $\sim 180 \mathrm{meV}$ complete gap once AFM is added to the scenario, hence the term "AFM gap." One important difference between the theoretical bulk calculation and the experimentally observed electronic states lies around the $M$ point, where the $\alpha$ and $\beta$ bands are observed to evolve up approaching $E_{F}$, making the circular electronlike shape of the $\beta$ band, while no bands are present within $E_{b}<0.4 \mathrm{eV}$ in the bulk calculation [red arrow in Fig. 8(c)]. This difference enables the fine spin texture of the $\beta$ contour, indicating strong surface modification of the bands resolved by ARPES.

Although the layered nature of $\mathrm{Sr}_{3} \mathrm{Ir}_{2} \mathrm{O}_{7}$ and the observed band dispersion (at higher binding energies) is similar to that of the bulk bands, a surface effect can take place due to the residual interactions between two $\mathrm{Ir}_{2} \mathrm{O}_{4}$ blocks. At the surface, the inversion symmetry is broken; the spin degeneracy is lifted due to the mutual effects of SOC and inversion symmetry breaking. Such spin splitting cannot be explained within the bulk band structure scenario; since the bulk crystal structure of $\mathrm{Sr}_{3} \mathrm{Ir}_{2} \mathrm{O}_{7}$ possesses inversion symmetry, bulk bands must be spin degenerate even if they are quasi two dimensional. The surface effects are thus important to the interpretation of our data reflecting the near-surface ground state of the iridate. Noticeably, the spin fine structure resolved for the $\beta$ band agrees qualitatively with a theoretical model where finite Coulomb $U$ and surface termination give rise to Rashba-like in-plane spin texture (Fig. 9). In the case of $U=0$, due to spin-orbit coupling (SOC) and inversion symmetry breaking, both the $\alpha$ and $\beta$ bands develop their surface counterpart which exhibit similar bulk band dispersion but finite spin splitting and a small degree of spin polarization. The directions of the spin are strictly in-plane $\left(S_{z}=0\right)$ and obey $E(k, \uparrow)=E(-k, \downarrow)$ due to time-reversal symmetry. When $U$ is turned on, AFM order is obtained self-consistently with finite staggered magnetic moments along the $z$ direction. The time-reversal symmetry is then broken and a finite $S_{z}$ component is obtained for each state (Fig. 9, right column). In our SR-ARPES measurements, we observed the in-plane fine spin texture and signature for the nonzero $S_{z}$ component of the $\beta$ band (Fig. 7). The presence of spin polarization supports the surface-dominated signal of the ARPES data, since this splitting is not expected in the bulk electronic structure. With all aspects of the data taken together,
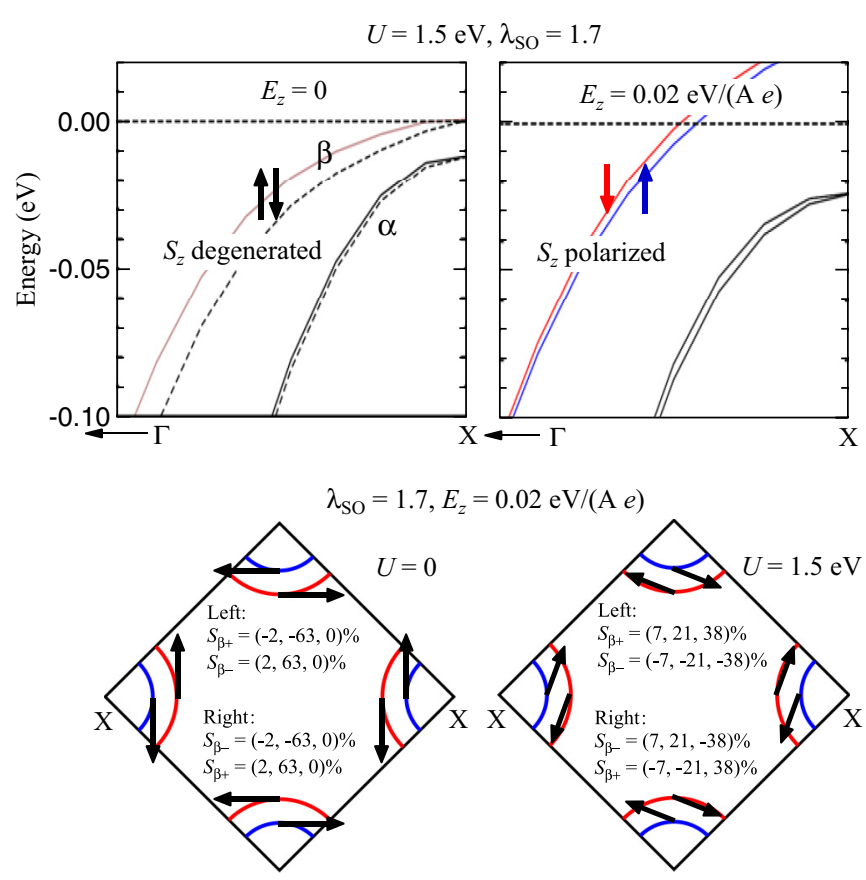

FIG. 9. (Color online) Spin texture of the $\beta$ band from slab calculations. Upper row: Slab calculation results for $\left(\lambda_{\mathrm{so}}, U, E_{z}\right)=$ $(1.7,1.5 \mathrm{eV}, 0)$ (left) and $(1.7,1.5 \mathrm{eV}, 0.02 \mathrm{eV} / \AA \cdot e)$ (right). Lower row: Slab calculation results (percentage of spin polarization) for $\left(\lambda_{\mathrm{so}}, U, E_{z}\right)=(1.7,0 \mathrm{eV}, 0.02 \mathrm{eV} / \AA \cdot e)$ (left) and $(1.7,1.5 \mathrm{eV}$, $0.02 \mathrm{eV} / \AA \cdot e$ ) (right). The in-plane Rashba-like spin polarization changes sign, while the out-of-plane Zeeman-like spin polarization emerges once a finite $U$ is introduced in the calculation.

our experimental observation and first-principles calculations suggest an interplay between SOC and the (bulk and/or surface) AFM order. Final-state photoelectron spin effects reported in strong spin-orbit coupled topological insulators are minimized here, since the spin-orbit splitting and effective coupling in this system is even weaker than that in gold.

\section{CONCLUSIONS}

The surface electronic ground state of $\mathrm{Sr}_{3} \mathrm{Ir}_{2} \mathrm{O}_{7}$, i.e., the $n=2$ member of the Ruddlesden-Popper iridate series $\mathrm{Sr}_{n+1} \mathrm{Ir}_{n} \mathrm{O}_{3 n+1}$, is distinct from that in $\mathrm{Sr}_{2} \mathrm{IrO}_{4}$ which realizes a $J=1 / 2$ Mott insulating state. Finite density of states is found at the Fermi level in $\mathrm{Sr}_{3} \mathrm{Ir}_{2} \mathrm{O}_{7}$, with a gaplike spectral weight suppression on the order of $40 \mathrm{meV}$. Spin-resolved ARPES data reveal a strong left-right imbalanced modulation on the surface, and find a spin fine structure in one of the bands, which likely results from on-site Coulomb interaction and bulk and/or surface antiferromagnetism. These observations are evident for a strong interplay between spin-orbit coupling, bandwidth, long-range magnetic order, as well as surface formation in $\mathrm{Sr}_{3} \mathrm{Ir}_{2} \mathrm{O}_{7}$. Our results provide insight into the quasiparticle interactions near the surface of this system.

\section{ACKNOWLEDGMENTS}

Work at Princeton and Princeton-led synchrotron-based measurements and the related theory at Northeastern University are supported by the Office of Basic Energy 
Sciences, U.S. Department of Energy (Grants No. DE-FG-02-05ER46200, No. AC03-76SF00098, and No. DE-FG02-07ER46352), and benefited from the allocation of supercomputer time at NERSC and Northeastern University's Advanced Scientific Computation Center. Work at Boston College is supported by NSF CAREER DMR-1056625. T.-R.C. is supported by the National Science Council and Academia Sinica, Taiwan, and would like to thank NCHC, CINC-NTU, and NCTS, Taiwan for technical support. The Advanced Light Source is supported by the Director, Office of Science, Office of Basic Energy Sciences, of the U.S. Department of Energy under Contract No. DE-AC02-05CH11231. The Stanford Synchrotron Radiation
Lightsource is supported by the U.S. Department of Energy under Contract No. DE-AC02-76SF00515. The Synchrotron Radiation Center is primarily funded by the University of Wisconsin-Madison with supplemental support from facility users and the University of Wisconsin-Milwaukee. N.A. is supported by NSF-DMR-1006492. We gratefully thank SungKwan Mo, Jonathan D. Denlinger, Donghui Lu, and Mark Bissen for instrumental support, and Vidya Madhavan for fruitful discussion. C.L. acknowledges Peng Zhang, Takeshi Kondo, and Adam Kaminski for the provision of data analysis software. M.Z.H. acknowledges Visiting Scientist support from LBNL and additional support from the A. P. Sloan Foundation.
[1] H. Y. Hwang, Y. Iwasa, M. Kawasaki, B. Keimer, N. Nagaosa, and Y. Tokura, Nat. Mater. 11, 103 (2012).

[2] A. F. Santander-Syro, O. Copie, T. Kondo, F. Fortuna, S. Pailhès, R. Weht, X. G. Qiu, F. Bertran, A. Nicolaou, A. Taleb-Ibrahimi, P. Le Fèvre, G. Herranz, M. Bibes, N. Reyren, Y. Apertet, P. Lecoeur, A. Barthélémy, and M. J. Rozenberg, Nature (London) 469, 189 (2011).

[3] S. Thiel, G. Hammerl, A. Schmehl, C. W. Schneider, and J. Mannhart, Science 313, 1942 (2006).

[4] J. E. Moore, Nature (London) 464, 194 (2010).

[5] S.-Y. Xu, M. Neupane, C. Liu, D. Zhang, A. Richardella, L. A. Wray, N. Alidoust, M. Leandersson, T. Balasubramanian, J. Sánchez-Barriga, O. Rader, G. Landolt, B. Slomski, J. H. Dil, J. Osterwalder, T.-R. Chang, H.-T. Jeng, H. Lin, A. Bansil, N. Samarth, and M. Z. Hasan, Nat. Phys. 8, 616 (2012).

[6] K. Ishizaka, M. S. Bahramy, H. Murakawa, M. Sakano, T. Shimojima, T. Sonobe, K. Koizumi, S. Shin, H. Miyahara, A. Kimura, K. Miyamoto, T. Okuda, H. Namatame, M. Taniguchi, R. Arita, N. Nagaosa, K. Kobayashi, Y. Murakami, R. Kumai, Y. Kaneko, Y. Onose, and Y. Tokura, Nat. Mater. 10, 521 (2011).

[7] D. Kim, S. Cho, N. P. Butch, P. Syers, K. Kirshenbaum, S. Adam, J. Paglione, and M. S. Fuhrer, Nat. Phys. 8, 459 (2012).

[8] G. Cao, Y. Xin, C. S. Alexander, J. E. Crow, P. Schlottmann, M. K. Crawford, R. L. Harlow, and W. Marshall, Phys. Rev. B 66, 214412 (2002).

[9] S. J. Moon, H. Jin, K. W. Kim, W. S. Choi, Y. S. Lee, J. Yu, G. Cao, A. Sumi, H. Funakubo, C. Bernhard, and T. W. Noh, Phys. Rev. Lett. 101, 226402 (2008).

[10] B. J. Kim, H. Jin, S. J. Moon, J.-Y. Kim, B.-G. Park, C. S. Leem, J. Yu, T. W. Noh, C. Kim, S.-J. Oh, J.-H. Park, V. Durairaj, G. Cao, and E. Rotenberg, Phys. Rev. Lett. 101, 076402 (2008).

[11] Q. Wang, Y. Cao, J. A. Waugh, S. R. Park, T. F. Qi, O. B. Korneta, G. Cao, and D. S. Dessau, Phys. Rev. B 87, 245109 (2013).

[12] P. D. C. King, T. Takayama, A. Tamai, E. Rozbicki, S. M. Walker, M. Shi, L. Patthey, R. G. Moore, D. Lu, K. M. Shen, H. Takagi, and F. Baumberger, Phys. Rev. B 87, 241106(R) (2013).
[13] B. M. Wojek, M. H. Berntsen, S. Boseggia, A. T. Boothroyd, D. Prabhakaran, D. F. McMorrow, H. M. Rønnow, J. Chang, and O. Tjernberg, J. Phys.: Condens. Matter 24, 415602 (2012).

[14] G. Jackeli and G. Khaliullin, Phys. Rev. Lett. 102, 017205 (2009).

[15] A. Shitade, H. Katsura, J. Kuneš, X.-L. Qi, S.-C. Zhang, and N. Nagaosa, Phys. Rev. Lett. 102, 256403 (2009).

[16] Y. Machida, S. Nakatsuji, S. Onoda, T. Tayama, and T. Sakakibara, Nature (London) 463, 210 (2010)

[17] R. Comin, G. Levy, B. Ludbrook, Z.-H. Zhu, C. N. Veenstra, J. A. Rosen, Yogesh Singh, P. Gegenwart, D. Stricker, J. N. Hancock, D. van der Marel, I. S. Elfimov, and A. Damascelli, Phys. Rev. Lett. 109, 266406 (2012).

[18] X. Wan, A. M. Turner, A. Vishwanath, and S. Y. Savrasov, Phys. Rev. B 83, 205101 (2011).

[19] B.-J. Yang and Y. B. Kim, Phys. Rev. B 82, 085111 (2010).

[20] F. Wang and T. Senthil, Phys. Rev. Lett. 106, 136402 (2011).

[21] J. P. Perdew, K. Burke, and M. Ernzerhof, Phys. Rev. Lett. 77, 3865 (1996).

[22] P. E. Blöchl, Phys. Rev. B 50, 17953 (1994); G. Kresse and D. Joubert, ibid. 59, 1758 (1999).

[23] G. Kresse and J. Hafner, Phys. Rev. B 48, 13115 (1993); G. Kresse and J. Furthmüller, Comput. Mater. Sci. 6, 15 (1996); Phys. Rev. B 54, 11169 (1996).

[24] M. A. Subramanian, M. K. Crawford, and R. L. Harlow, Mater. Res. Bull. 29, 645 (1994).

[25] S. Boseggia, R. Springell, H. C. Walker, A. T. Boothroyd, D. Prabhakaran, D. Wermeille, L. Bouchenoire, S. P. Collins, and D. F. McMorrow, Phys. Rev. B 85, 184432 (2012).

[26] Slightly different crystal structures of $\mathrm{Sr}_{3} \mathrm{Ir}_{2} \mathrm{O}_{7}$ are reported by different groups. See, e.g., Ref. [24], Ref. [8], and H. Matsuhata et al., J. Solid State Chem. 177, 3776 (2004) for details. Here we use the structure reported by Cao et al. (Ref. [8]). Note that although Cao et al. reported an orthorhombic crystal structure, its $a$ and $b$ lattice constants are so close to each other that we cannot resolve the difference using ARPES. Thus, all Brillouin zones used in this paper appear to be tetragonal-like.

[27] C. Dhital, S. Khadka, Z. Yamani, C. de la Cruz, T. C. Hogan, S. M. Disseler, M. Pokharel, K. C. Lukas, W. Tian, C. P. Opeil, Z. Wang, and S. D. Wilson, Phys. Rev. B 86, 100401(R) (2012). 
[28] Y. Okada, D. Walkup, H. Lin, C. Dhital, T.-R. Chang, S. Khadka, W. Zhou, H.-T. Jeng, M. Paranjape, A. Bansil, Z. Wang, S. D. Wilson, and V. Madhavan, Nat. Mater. 12, 707 (2013).

[29] J. W. Kim, Y. Choi, Jungho Kim, J. F. Mitchell, G. Jackeli, M. Daghofer, J. van den Brink, G. Khaliullin, and B. J. Kim, Phys. Rev. Lett. 109, 037204 (2012).

[30] Y.-D. Chuang, A. D. Gromko, D. S. Dessau, T. Kimura, and Y. Tokura, Science 292, 1509 (2001).
[31] P. Zhang, P. Richard, T. Qian, Y. M. Xu, X. Dai, and H. Ding, Rev. Sci. Instrum. 82, 043712 (2011).

[32] C. Liu, T. Kondo, R. M. Fernandes, A. D. Palczewski, E. D. Mun, N. Ni, A. N. Thaler, A. Bostwick, E. Rotenberg, J. Schmalian, S. L. Bud'ko, P. C. Canfield, and A. Kaminski, Nat. Phys. 6, 419 (2010).

[33] F. Meier, J. H. Dil, and J. Osterwalder, New J. Phys. 11, 125008 (2009). 